\title{
Ferroelectric Electron Holography
}

Hannes Lichte, Marianne Reibold, Karin Brand and Michael Lehmann

Institute of Applied Physics, Dresden University, D 01062 Dresden, Germany, www.triebenberg.de

Conventional TEM-investigation of ferroelectric structures has been a difficult task since decades. To assess the facilities of electron holography for the determination of the electric polarisation in ferroelectrics, a simple dipole model was set up [1]. It turns out that by interaction of the electron wave with an array of equally oriented dipoles - like in a ferroelectric domain - the electron phase is modulated in two aspects: First, each dipole modulates the wave microscopically according to the positive and negative charges in each unit cell; second, there is a mesoscopic phase shift nearly linearly increasing in direction of the polarisation (fig.1). These phase shifts arise only from the "inplane"-components of polarisation perpendicular to the electron beam, whereas the electron wave is not affected by the "out-of-plane" components along the electron beam; Spence et al. already showed this theoretically [2]. The mesoscopic phase modulation can be computed as

$$
\varphi(\vec{r})=\frac{\sigma}{\varepsilon} \iint_{t}\left[\int_{0}^{\vec{r}} \vec{P}_{i p} d \vec{r}\right] d z
$$

with $\sigma$ the interaction constant of the microscope, $\varepsilon=\varepsilon_{0} \varepsilon_{r}$ the dielectric constant, and $t$ the object thickness; $\vec{P}_{i p}$ is the in-plane component of polarisation. Assuming constant polarisation over thickness $t$, this amounts to

$$
\varphi(\vec{r})=\frac{\sigma t}{\varepsilon} \int_{0}^{\vec{r}} \vec{P}_{i p} d \vec{r}
$$

Consequently, the in-plane component $\vec{P}_{i p}$ can be determined from the phase image by means of

$$
\vec{P}_{i p}=\frac{\varepsilon}{\sigma t} \operatorname{grad}[\varphi(\vec{r})] \text {. }
$$

Using our CM200FEG-ST/Lorentz electron microscope with $\sigma=0.0073 /(\mathrm{V} \mathrm{nm})$, we took holograms of $\mathrm{BaTiO}_{3}$ with Lorentz-lens covering a field of view of about $800 \mathrm{~nm}$ in square. Assuming an object thickness of $t=50 \mathrm{~nm}$, for $\mathrm{BaTiO}_{3}\left(\vec{P}_{i p}=\vec{P}=26 \mu \mathrm{C} / \mathrm{cm}^{2}, \varepsilon_{r} \approx 1,700\right)$ a phase gradient of the order of $2 \pi / 1,000 \mathrm{rad} / \mathrm{nm}$ can be expected. In the phase images, we found significant phase modulations over domains (fig.2); the surface plot of the phase image allows visual inspection in terms of polarisation; the phase gradient is more accurately displayed as an arrow plot. To determine also the out-of plane components, one has to tilt the specimen around a well-known, arbitrary in-plane axis; from these two phase images the distribution of total polarisation $\vec{P}$ can be determined in strength and orientation.

\section{References}

[1] H. Lichte, Cryst.Res.Technol. 35(2000), 887

[2] J.C.H. Spence, J.M. Cowley and J.M. Zuo, Appl.Phys.Lett 62(19), 2446

We thank Prof. Dietrich Schulze for support and discussions, as well as the Deutsche Forschungsgemeinschaft for funding this project. 


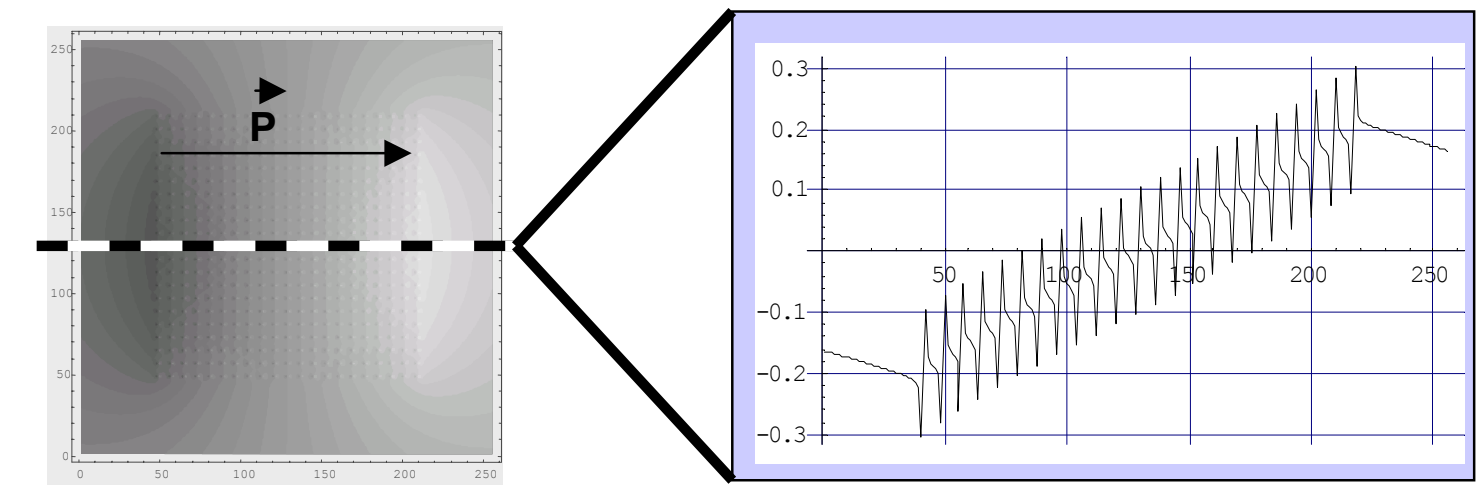

Fig.1 Phase shift produced by a 2D-model of a ferroelectric domain made up by a mono-layer of $21 * 21$ dipoles (left); the line scan (right) shows that - in addition to the modulation by the single dipoles - a far-reaching phase slope of the electron wave over the whole domain is found.
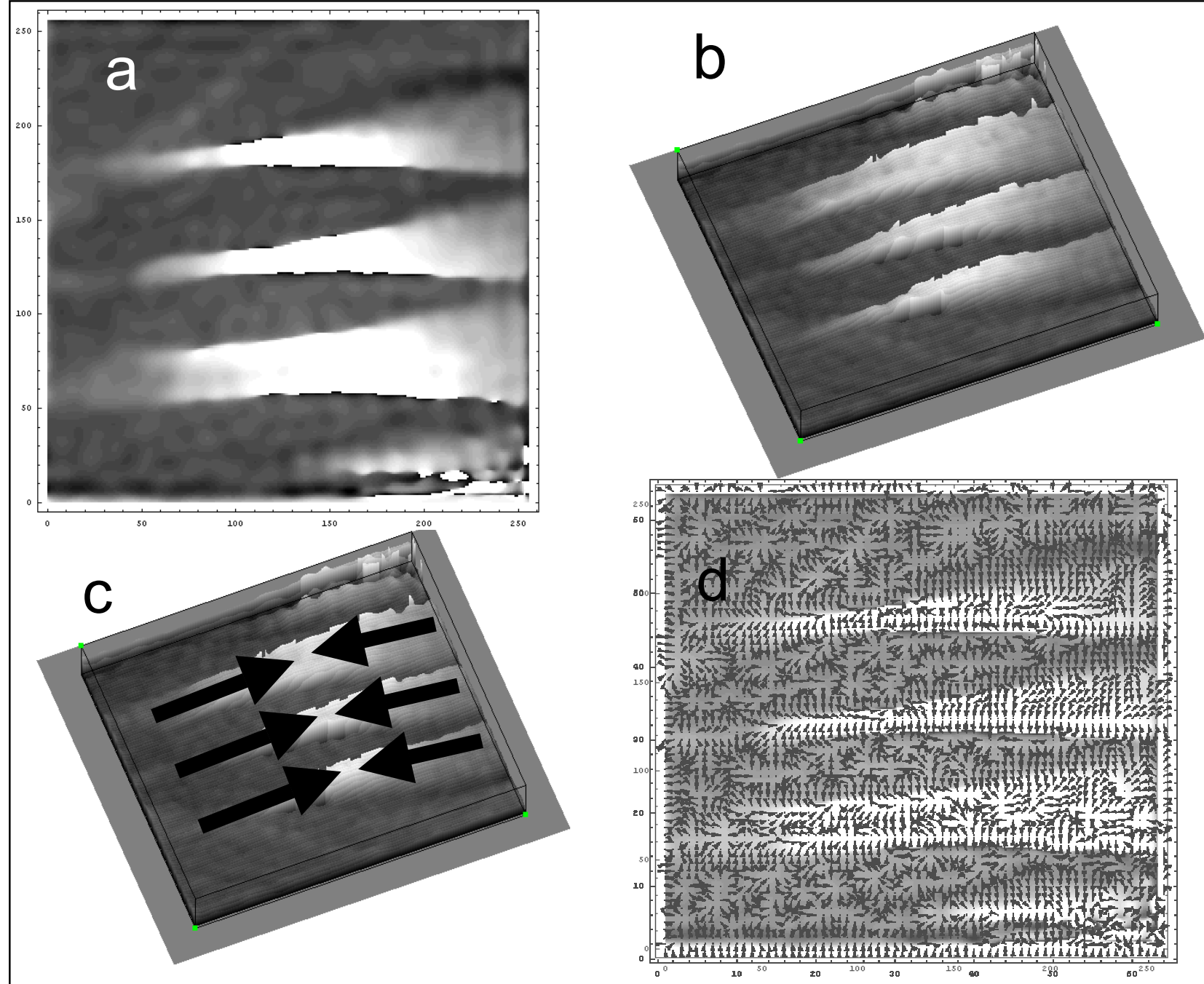

Fig.2 Phase in ferroelectric domains in $\mathrm{BaTiO}_{3}(\mathrm{a})$, phase surface (b) suggesting polarisation (c), and phase gradient as arrow plot showing polarisation distribution. No in-plane polarisation is found in the dark areas which are virtually flat. 